The Egyptian Journal of Hospital Medicine (July 2019) Vol. 76 (5), Page 4182-4188

\title{
Macular Thickness Asymmetry Measurements in Relatives of Primary Open Angle Glaucoma Patients
}

\author{
Sherien R. Ramadan*, Mona M. Aly, Samia H. El Ashry \\ Department of Ophthalmology, Faculty of Medicine (For Girls), Al-Azhar University \\ *Corresponding Author: Sherien R Ramadan; Mobile: 00201026822888; email: sherien.roshdy1 @ gmail.com
}

\begin{abstract}
Background: A family history of glaucoma was found to carry a relative risk of 2.1 times for being associated with at least possible primary open angle glaucoma (POAG) in middle aged and older individuals. Around half of all POAG patients have a positive family history and their first degree relatives have an approximately 9 -fold increased risk of developing glaucoma.

Objective: the aim of the present study was to demonstrate possible early macular alterations in young relatives of POAG patients using spectral domain optical coherence tomography (SD-OCT) posterior pole asymmetry analysis (PPAA) map. Patients and methods: in a prospective comparative study; 40 subjects were classified into 2 groups: group of young healthy relatives of POAG patients and another group of young healthy subjects as control. All participants had OCT of the PPAA, retinal nerve fiber layer (RNFL), ganglion cell complex (GCC) and hemi-field macular thickness.

Results: There was no statistically significant difference between the relative and control group in aspect of RNFL, macular hemi-fields and PPAA measurements (P-value >0.05).

Regarding GCC thickness, there were statistically significant differences between both groups in the superior, inferior and nasal thickness measurements.

Conclusion: Young healthy relatives of POAG patients didn't show any early glaucomatous damage when compared with controls except for GCC thickness which showed statistically significant decrease.

Keywords: Primary open angle glaucoma, Optical coherence tomography, Posterior pole asymmetry analysis, Retinal nerve fiber layer, Ganglion cell complex.
\end{abstract}

\section{INTRODUCTION}

Glaucoma is a group of chronic progressive neuropathies that cause morphological changes to the optic nerve head $(\mathrm{ONH})$ and retinal nerve fiber layer (RNFL) that are associated with visual field defects ${ }^{(\mathbf{1})}$. Primary open angle glaucoma (POAG) is the most common type of glaucoma and globally considered the most common cause of irreversible blindness, with an estimated 70 million affected individuals ${ }^{(2)}$. POAG can be defined as progressive characteristic optic disc changes which are usually associated with corresponding visual field defects ${ }^{(3)}$.

The presence of a family history of glaucoma was found to have a relative risk of 2.1 times for being correlated with at least possible OAG. However, the relative importance of family history may vary according to the closeness of relationship of a patient to an affected family member (first, second or even third degree). Around half of all POAG patients have a positive family history and their first degree relatives (parents, siblings or children) have an approximately 9fold increased risk of developing glaucoma ${ }^{(4)}$.

Spectral-domain optical coherence tomography (SD-OCT) is considered one of the most promising image modalities in evaluating glaucoma. Heidelberg Engineering (Spectralis SD-OCT, Heidelberg, Germany) customized the most recent retinal thickness protocol to obtain retinal thickness measurement of the central of the posterior pole ${ }^{(5)}$. The posterior pole retinal thickness map is a color-coded map that provides mean retinal thickness value of grid centered on the foveal pit. The grid is positioned symmetrically to the fovea-disc axis ${ }^{(6)}$.

The current study was conducted to demonstrate possible early macular alterations in young relatives of primary open angle glaucoma patients using Spectralis SD-OCT posterior pole asymmetry analysis map.

\section{PATIENT AND METHODS}

This prospective comparative study included forty subjects classified into two groups; group I of young healthy relatives of POAG patients and another group II of young healthy subjects as control. Their age ranged from 17-40 years.

Ethical approval:

An informed written consent from all the subjects and the approval of the Medical Ethics Committee of Al-Azhar University were obtained. It was done adhering to the principles of the Declaration of Helsinki guidelines.

Inclusion criteria included:

- Young relatives of POAG patients (having at least one POAG relative $\left(1^{\text {st }}\right.$ or $2^{\text {nd }}$ degree $)$ ) with spherical equivalent between -1.5 and +1.5 diopters with best corrected visual acuity (BCVA) 20/20 in both eyes.

- Normal age-matched healthy adults with a normal eye examination and a spherical equivalent between -1.5 and +1.5 diopters with BCVA 20/20 in both eyes. 
- Bilateral intraocular pressure (IOP) $<21 \mathrm{~mm} \mathrm{Hg}$ with no history of elevated IOP.

\section{Exclusion criteria included:}

- Ocular diseases (e.g. corneal irregularity, dry eye, amblyopia, anisometropia > 1.00 diopters, glaucoma, retinal abnormalities, etc.).

- Any condition preventing adequate examination of the fundus (e.g. ptosis, dense corneal opacities or lens opacities).

- Active infection of the anterior or posterior segments of the eye.

- Previous intraocular surgery, laser procedure or ocular trauma.

- History of neuro-ophthalmological disease (optic neuritis, multiple sclerosis, etc.).

- Any systemic diseases e.g. diabetes mellitus, hypertension, etc.

- Medications that might affect the eye.

- High ametropia.

All patients had full ophthalmological examination including:

○ Visual acuity and refraction.

- Slit lamp biomicroscopy.

- IOP measurement by applanation tonometer.

- Examination of posterior segment.

Optical Coherence Tomography Measurements:

Spectralis SD-OCT (Heidelberg Engineering, Heidelberg, Germany) assessment of the macular thickness measurements in both eyes of each person using the posterior pole asymmetry scan protocol, peripapillary RNFL measurements and macular ganglion cell complex (GCC) thickness measurement were performed. The GCC consists of the three inner most retinal layers: the RNFL, the ganglion cell layer and the inner plexiform layer. For GCC analysis, the built in macular star shaped scan was used to measure the GCC using manual caliber tools provided by Spectralis software (Heidelberg Engineering, Heidelberg, Germany), at a horizontal and vertical placement of $600 \mu \mathrm{m}$ temporal, nasal, superior and inferior from the center of the fovea to achieve standardization. The OCT scans were excluded if the image quality was less than 25 decibels. All study eyes were not dilated before image acquisition. Participants in this study were instructed to fixate on the intrinsic fixation target during the whole process of OCT scanning.

\section{Statistical analysis:}

Data were collected, revised, coded and entered to the Statistical Package for Social Science (IBM SPSS) version 23(IBM Corp., Armonk, New York, USA). The quantitative data were presented as mean, standard deviations (SD) and ranges. Also qualitative variables were presented as number and percentages. The comparison between groups regarding qualitative data was done by using Chi-square $(\chi 2)$ test. The comparison between two groups regarding quantitative data and parametric distribution was done by using Independent $t$-test while with non-parametric distribution was done by using Mann-Whitney test. Pvalue $>0.05$ : was considered non-significant (NS); Pvalue $<0.05$ Significant $(\mathrm{S})$ and $\mathrm{P}$-value $<0.01$ : Highly significant (HS).

\section{RESULTS}

Demographic and Clinical characteristics of the Study Population (Table 1 and Figure 1):

There was statistically non-significant difference between groups according to demographic data.

Table (1): Comparison between the two groups according to demographic data

\begin{tabular}{|l|l|l|l|l|c|}
\hline \multicolumn{2}{|c|}{} & Group I & Group II & \multirow{2}{*}{ P-value } & \multirow{2}{*}{ Sig. } \\
\cline { 3 - 4 } \multicolumn{2}{|c|}{$\begin{array}{l}\text { Age } \\
\text { (Years) }\end{array}$} & Mea $=\mathbf{2 0}$ & No. $=\mathbf{2 0}$ & & \\
\cline { 2 - 4 } Sex & Range & $26.55 \pm 6.70$ & $29.15 \pm 3.80$ & \multirow{2}{*}{$>0.05$} & \multirow{2}{*}{ NS } \\
& Female & $17-40$ & $19-39$ & & \\
\cline { 2 - 4 } & Male & $4(20.0 \%)$ & $8(40.0 \%)$ & \multirow{2}{*}{ NS } \\
\hline
\end{tabular}


Degree of Relativity

口1st a2nd

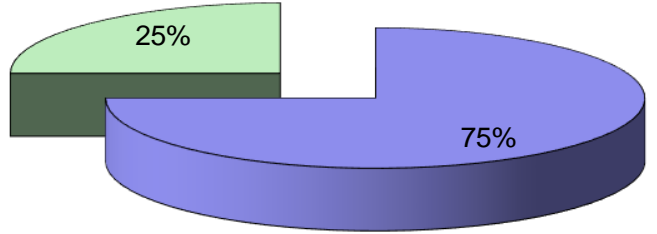

Figure (1): The degree of relativity distribution in group $I$.

The mean spherical equivalent (SE), IOP and vertical Cup/Disc ratio of both groups were shown in Table (2); there was statistically non-significant difference between the two groups in SE and IOP; although the mean vertical Cup/Disc ratio was slightly higher in the relatives group, the difference was statistically non-significant $(P>0.05)$.

Table (2): Comparison between both groups with respect to SE, IOP and Vertical C/D ratio

\begin{tabular}{|c|l|l|l|l|c|}
\hline \multicolumn{2}{|c|}{} & Group I & Group II & \multirow{2}{*}{ P-value } & \multirow{2}{*}{ Sig. } \\
\cline { 3 - 4 } & No. $=\mathbf{4 0}$ & No. $=\mathbf{4 0}$ & & \\
\hline \multirow{2}{*}{ SE (Diopter) } & Mean \pm SD & $-0.14 \pm 0.50$ & $-0.08 \pm 0.45$ & \multirow{2}{*}{$>0.05$} & \multirow{2}{*}{ NS } \\
\cline { 2 - 4 } & Range & $-1.25-1$ & $-0.75-0.75$ & & \\
\hline \multirow{2}{*}{ IOP(mmHg) } & Mean \pm SD & $14.13 \pm 1.54$ & $14.48 \pm 1.60$ & \multirow{2}{*}{$>0.05$} & \multirow{2}{*}{ NS } \\
\cline { 2 - 4 } & Range & $11-17$ & $12-18$ & & \multirow{2}{*}{$>0.05$} \\
\hline \multirow{2}{*}{ Vertical C/D } & Mean \pm SD & $0.28 \pm 0.18$ & $0.26 \pm 0.14$ & \multirow{2}{*}{ NS } \\
\cline { 2 - 4 } & Range & $0.1-0.7$ & $0.1-0.6$ & & \\
\hline
\end{tabular}

$\mathrm{SE}=$ spherical equivalent, $\mathrm{IOP}=$ intraocular pressure, $\mathrm{C} / \mathrm{D}=$ cup to disc ratio

OCT Measurements of the Study Population:

- Peripapillary RNFL Measurements (Table 3 and Figure 2):

The mean peripapillary RNFL thickness was $104.78 \pm 8.89 \mu \mathrm{m}$ in the relatives group (Group I) and $104.73 \pm 8.10$ $\mu \mathrm{m}$ in the control group (Group II). A statistically non-significant difference was observed between the two groups according to the mean peripapillary RNFL thickness (P-value $=0.979)$. Segmental peripapillary RNFL thickness (Inferior, Superior, Nasal and Temporal) are shown in Table (3). There were statistically non-significant differences in the segmental peripapillary RNFL thickness of the quadrants between the 2 groups.

Table (3): Peripapillary RNFL thickness values in relatives and control groups

\begin{tabular}{|c|c|c|c|c|c|}
\hline \multirow{2}{*}{\multicolumn{2}{|c|}{ RNFL Thickness $(\mu \mathrm{m})$}} & Group I & Group II & \multirow[t]{2}{*}{ P-value } & \multirow[t]{2}{*}{ Sig } \\
\hline & & No. $=40$ & No. $=40$ & & \\
\hline \multirow[t]{2}{*}{ Mean RNFL thickness } & Mean \pm SD & $104.78 \pm 8.89$ & $104.73 \pm 8.10$ & \multirow[t]{2}{*}{$>0.05$} & \multirow[t]{2}{*}{ NS } \\
\hline & Range & $90-130$ & $91-120$ & & \\
\hline \multirow[t]{2}{*}{ Inferior quadrant } & Mean \pm SD & $133.88 \pm 13.40$ & $138.08 \pm 19.89$ & \multirow[t]{2}{*}{$>0.05$} & \multirow[t]{2}{*}{ NS } \\
\hline & Range & $102-165$ & $108-180$ & & \\
\hline \multirow[t]{2}{*}{ Superior quadrant } & Mean \pm SD & $130.05 \pm 15.66$ & $130.25 \pm 15.08$ & \multirow[t]{2}{*}{$>0.05$} & \multirow[t]{2}{*}{ NS } \\
\hline & Range & $99-173$ & $101-164$ & & \\
\hline \multirow[t]{2}{*}{ Nasal quadrant } & Mean \pm SD & $79.35 \pm 14.51$ & $75.55 \pm 11.83$ & \multirow[t]{2}{*}{$>0.05$} & \multirow[t]{2}{*}{ NS } \\
\hline & Range & $52-107$ & $53-101$ & & \\
\hline \multirow[t]{2}{*}{ Temporal quadrant } & Mean \pm SD & $74.40 \pm 11.10$ & $75.08 \pm 8.27$ & \multirow[t]{2}{*}{$>0.05$} & \multirow[t]{2}{*}{ NS } \\
\hline & Range & $60-111$ & $60-103$ & & \\
\hline
\end{tabular}

$\mathrm{RNFL}=$ retinal nerve fiber layer 


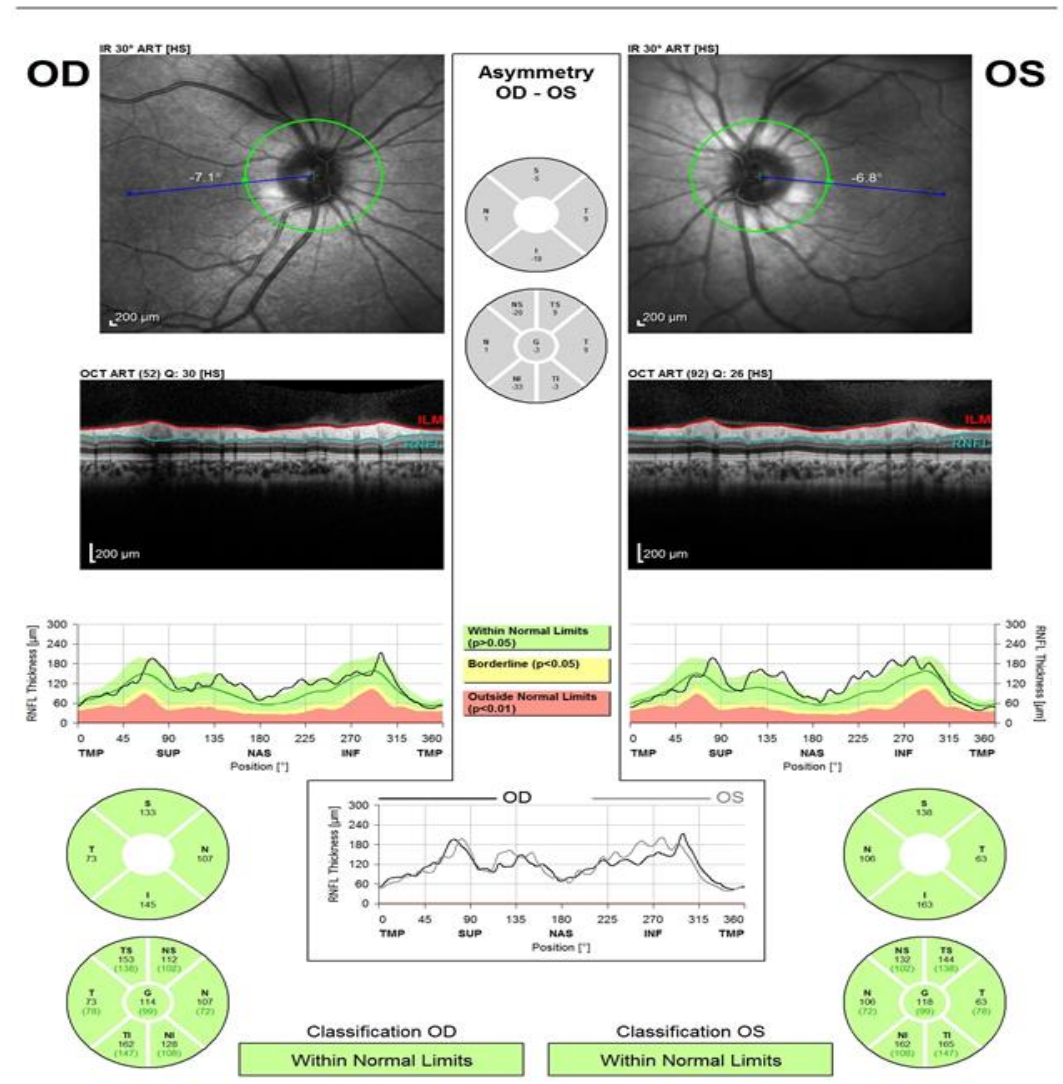

Figure (2): RNFL thickness measurements of a relative from group I measured by Spectralis-OCT.

- Macular Thickness Measurements (Table 4 and Figure 3):

The superior and whole macular thickness measurements were slightly higher in the relatives group (Group I) than the control group (Group II); however this was statistically non-significant. The inferior hemi-field macular thickness measurements were slightly higher in the control group (Group II) than in the relatives group (Group I); however this was statistically non-significant.

The asymmetry between superior and inferior hemifield macular thickness was calculated by subtracting the two corresponding values. PPAA revealed statistically non-significant difference between the groups.

Table (4): Mean Macular Thickness Measurements of Whole macula, Superior and Inferior hemi-fields

\begin{tabular}{|l|l|l|l|l|c|}
\hline \multicolumn{2}{|c|}{$\begin{array}{c}\text { Mean Macular Thickness } \\
\text { Measurements }(\boldsymbol{\mu m})\end{array}$} & Group I & Group II & \multirow{2}{*}{ P-value } & \multirow{2}{*}{ Sig. } \\
\cline { 2 - 4 } & Mean \pm SD & $290.73 \pm 10.13$ & $289.95 \pm 12.48$ & \multirow{2}{*}{$>0.05$} & \multirow{2}{*}{ NS } \\
\cline { 2 - 4 } Whole Macula & Range & $271-312$ & $270-317$ & & \\
\hline \multirow{2}{*}{ Superior hemi-field } & Mean \pm SD & $291.18 \pm 10.00$ & $290.23 \pm 12.84$ & \multirow{2}{*}{$>0.05$} & NS \\
\cline { 2 - 4 } & Range & $272-309$ & $270-316$ & & \multirow{2}{*}{ NS } \\
\hline \multirow{2}{*}{ Inferior hemi-field } & Mean \pm SD & $282.68 \pm 34.01$ & $289.60 \pm 12.73$ & \\
\cline { 2 - 4 } & Range & $141-316$ & $267-318$ & & \\
\hline
\end{tabular}



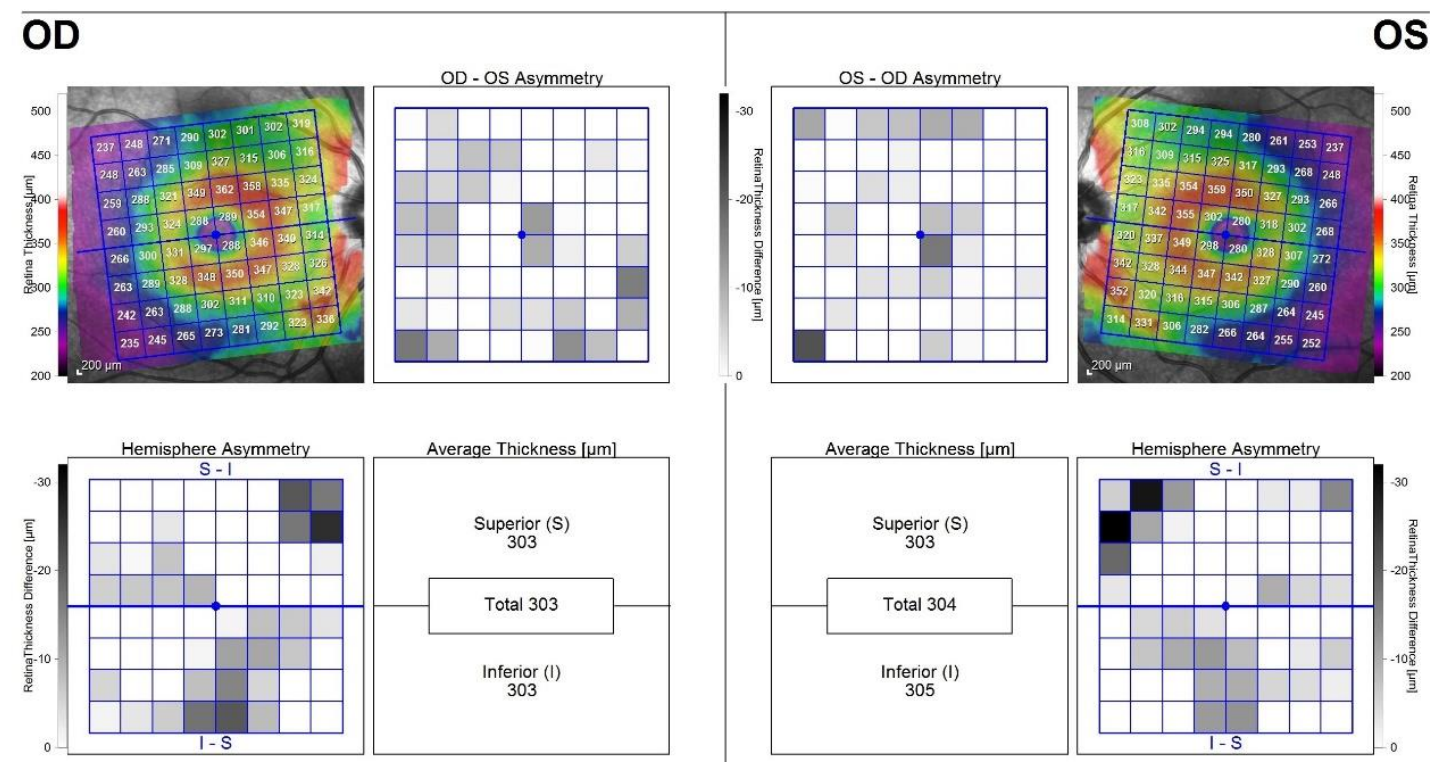

Figure (3): PPAA map of a relative from group I as provided by Spectralis-OCT.

\section{- Ganglion Cell Complex Measurements (Table 5 and Figure 4):}

There was statistically significant difference between the two groups with respect to the GCC thickness measurements superiorly, inferiorly and nasally while it was statistically non-significant temporally; with the nasal and inferior thickness measurements differences being highly significant.

Table (5): Ganglion cell complex measurements in both groups

\begin{tabular}{|c|c|c|c|c|c|}
\hline \multirow{2}{*}{\multicolumn{2}{|c|}{ GCC Thickness ( $\mu \mathrm{m})$}} & Group I & Group II & \multirow[t]{2}{*}{ P-value } & \multirow[t]{2}{*}{ Sig. } \\
\hline & & No. $=40$ & No. $=40$ & & \\
\hline \multirow[t]{2}{*}{ Superior Thickness } & Mean \pm SD & $91.83 \pm 7.38$ & $95.58 \pm 9.23$ & \multirow[t]{2}{*}{0.048} & \multirow[t]{2}{*}{$\mathbf{S}$} \\
\hline & Range & $78-105$ & $79-124$ & & \\
\hline \multirow[t]{2}{*}{ Inferior Thickness } & Mean \pm SD & $87.98 \pm 6.27$ & $93.75 \pm 9.32$ & \multirow[t]{2}{*}{0.002} & \multirow[t]{2}{*}{ HS } \\
\hline & Range & $77-99$ & $77-119$ & & \\
\hline \multirow[t]{2}{*}{ Nasal Thickness } & Mean \pm SD & $74.93 \pm 4.13$ & $86.90 \pm 8.13$ & \multirow[t]{2}{*}{0.001} & \multirow[t]{2}{*}{ HS } \\
\hline & Range & $67-83$ & $69-103$ & & \\
\hline \multirow[t]{2}{*}{ Temporal Thickness } & Mean \pm SD & $87.60 \pm 5.14$ & $90.93 \pm 10.35$ & \multirow[t]{2}{*}{$>0.05$} & \multirow[t]{2}{*}{ NS } \\
\hline & Range & $72-96$ & $71-124$ & & \\
\hline
\end{tabular}

GCC: Ganglion Cell Complex

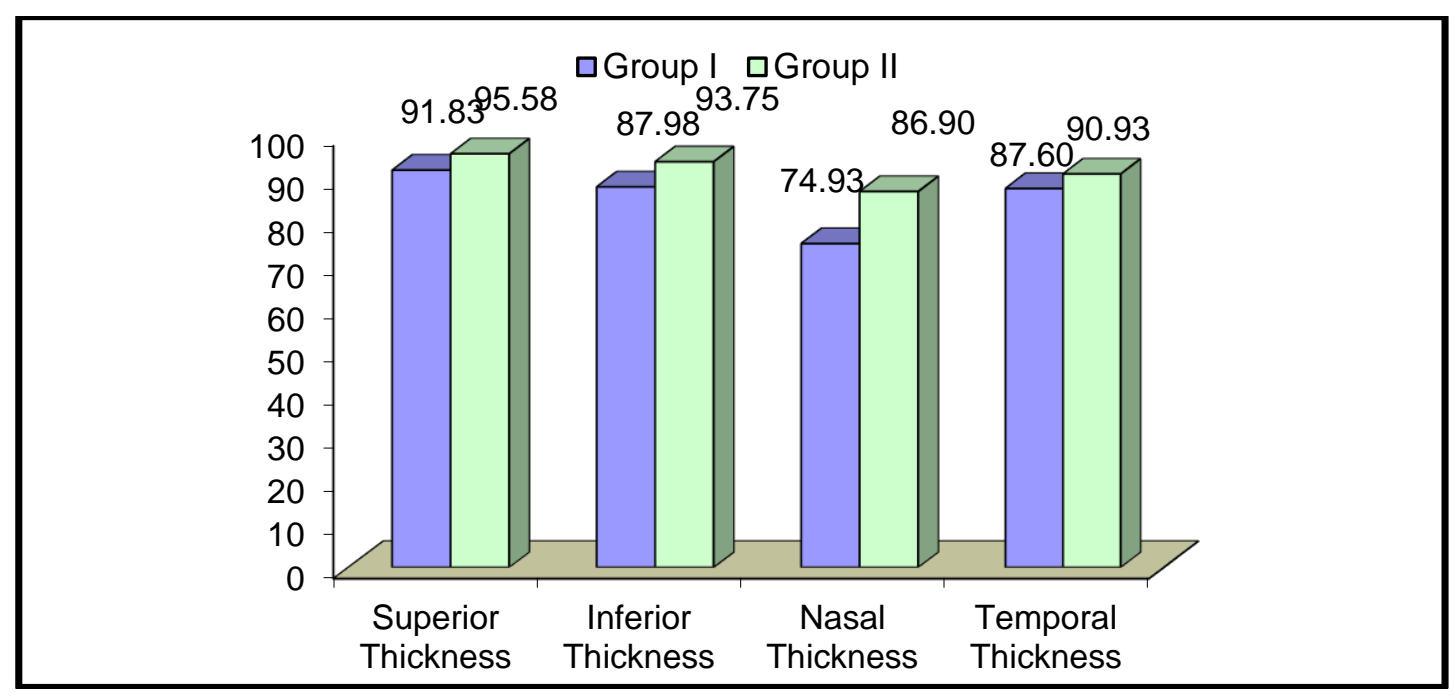

Figure (4): Ganglion cell complex measurements in both groups

There were no significant differences between first and second degree relatives of POAG patients with respect to the mean RNFL, PPAA and GCC thicknesses (P-value >0.05). 
ejhm.journals.ekb.eg

\section{DISCUSSION}

Glaucoma is the second most common cause of blindness that causes irreversible blindness. The World Health Organization has estimated that $12.3 \%$ of the 37 million people blind worldwide is attributed to glaucoma (7). POAG is the most common type of glaucoma with a higher prevalence in individuals of African ancestry ${ }^{(8)}$. While open-angle glaucoma is a chronic insidious process, patients are often unaware of their disease until vision loss has progressed significantly, known as the "sneak thief of sight". Early diagnosis remains a challenge given the insidious nature of the onset of this process and, therefore, formal ophthalmologic evaluation of any patient with risk factors is critical for prompt detection ${ }^{(\mathbf{9})}$.

Family history is a definite risk factor. Heritable susceptibility has been shown a percentage of $10-20 \%$ of glaucomatous patients have a positive family history. Studies found that family history of glaucoma in a sibling is a greater risk factor than glaucoma in a parent. The relative risk of having glaucoma was found to be increased 3.7-fold for individuals who have siblings with POAG ${ }^{(\mathbf{1 0})}$. The emerging view is that POAG is a complex, multifactorial disease resulting from interactions between several genetic and environmental factors ${ }^{(11)}$.

Based on the above considerations, in the present study, we evaluated 20 young healthy relatives of POAG patients of first and second degrees; however the study showed that the characteristic glaucomatous damage indicators including peripapillary RNFL thickness, IOP and PPAA were similar in young healthy relatives of POAG and healthy controls. Young adults were only included in the study to eliminate the age-related risk of glaucoma since age is an important factor in the occurrence of POAG.

It is now easier to detect glaucoma early with the development of SD-OCT and its new software programs as Khanal et al. ${ }^{(12)}$ stated that the recent introduction of SD-OCT technology provides real time, rapid, high resolution in vivo scans of ocular tissues and thus facilitates the assessment of RNFL as well as macular thickness. Normative OCT measurements in healthy population have been previously established as well as consistently shown that both peripapillary RNFL thickness and macular thickness are lower in glaucomatous eyes.

Investigating the importance of macular thickness measurements for early diagnosis of glaucomatous damage was done by a lot of studies. Several studies have shown that early glaucomatous diagnostic ability of macular thickness measurements was generally lower than that of peripapillary RNFL thickness measurements highlighting the importance of RNFL thickness measurements in early diagnosis of glaucoma (13-15). Sullivan-Mee et al. ${ }^{(16)}$ stated that although prior studies suggest that both RNFL thickness and macular thickness are thinner in eyes with glaucoma, RNFL thickness was found to be more capable of diagnosing glaucomatous damage than macular thickness. However when intra-eye macular thickness asymmetry is used instead of measured macular thickness. As, when Um and associates ${ }^{(17)}$ divided macular thickness measures into corresponding superior and inferior macular thickness zones and then evaluated symmetry between these zones, it was reported that the asymmetry in thickness between corresponding superior and inferior macular zones was more sensitive than RNFL thickness for detecting early POAG.

Yamashita et al. $^{(18)}$ stated that posterior pole asymmetry analysis (PPAA) by OCT was introduced for diagnosing glaucoma and is expected to improve the accuracy of diagnosing glaucoma. Its diagnostic accuracy was reported to be similar to that obtained by RNFL thickness circle scans.

In the current study, the superior and whole macular thickness measurement were slightly higher in the relatives group than the control group while the inferior hemi-field macular thickness measurements were slightly higher in the control group than in the relatives group; however no statistically significant difference was found while the peripapillary RNFL thickness values were similar in both groups. Also the asymmetries in macular thickness in each eye and between both eyes were not statistically different between the relatives and control group. Since there was no evidence of glaucoma in any of the participants this result does not indicate the superiority of macular thickness analysis over the peripapillary RNFL thickness analysis in early diagnosis of glaucoma.

The measurement of GCC thickness has emerged as a new diagnostic parameter as Yang et $\boldsymbol{a l} .{ }^{(19)}$ stated that OCT measurements of macular structures such as macular ganglion cell complex (mGCC) may be useful for differentiating healthy and glaucomatous eyes. Macular GCC was reported to have similar diagnostic performance to peripapillary RNFL, however, measurement of peripapillary RNFL was suggested to be better. Therefore, in the current study, we measured the GCC thickness and compared the relatives with the control group; the GCC measurements were thinner in the study group in the nasal, inferior and superior thickness measurements than in the control group and this was statistically significant. The previously mentioned result was in agreement with Rolle et $\boldsymbol{a l} .^{(20)}$ who reported that the eyes of subjects with positive family history have significantly thinner GCC than normal eyes. However since it was measured manually using the caliber tools provided by the Heidelberg software, it is possible that there would be a different outcome if another measurement technique was used.

In population screening studies, there is high number of glaucoma diagnosis in relatives of POAG patients (2122). In the present study, we didn't diagnose glaucoma 
neither in any of the relatives nor in controls, may this be due to that fact that our participants are younger than 40 years and the POAG most commonly occurs after 40 years. The participants had same IOP, RNFL however different GCC measurements. Also the PPAA notes no statistically significant difference.

The present study has its limitations; first we didn't measure the central corneal thickness, also although the GCC was measured at a more than single point it was measured manually and not automated. Also the sample size of the study is considered to be small, more participants of relatives of POAG needed to be screened in order to get more accurate results. Lastly, it would be beneficial to perform more functional tests to get additional parameters such as pattern electroretinogram which reflects retinal ganglion cell damage.

\section{CONCLUSION}

In conclusion, no glaucomatous damage was detected in any of the young healthy relatives of POAG participated in the study after measuring PPAA and peripapillary RNFL thickness. Also IOP and vertical cup to disc ratios were similar in both groups. Macular thickness in the inferior hemi-field was not the same although the difference was insignificant. GCC measurements were different but more accurate measuring should be done. As expected, age seems to be the main determinant in the occurrence of glaucomatous damage in the relatives of POAG patients. Further studies that would determine the macular thickness asymmetry with time need to be done.

Financial support and sponsorship: Nil.

Conflicts of interest: There are no conflicts of interest.

\section{REFERENCES}

1. Alluwimi MS, Swanson WH, King BJ (2018): Identifying glaucomatous damage to the macula. Optom Vis Sci., 95(2): 96105.

2. O'Brien C, Butt Z (1999): Blood flow velocity in the peripheral circulation of glaucoma patients. Ophthalmologica, 213:150-3.

3. Vegini F, Figueiroa-Filho NF, Lenci RF et al. (2008): Prevalence of open angle glaucoma in accompanying first degree relatives of patients with glaucoma. Clinics (Sao Paulo), 63(3):329-332.

4. McMonnies CW (2017): Glaucoma history and risk factors. J Optom., 10(2): 71-78.

5. Altan C, Arman BH, Arici M et al. (2019): Normative posterior pole asymmetry analysis data in healthy Caucasian population. Eur J Ophthalmol., 29(4):386-393.

6. Jacobsen AG, Bendtsen MD, Vorum H et al. (2015): Normal value ranges for central retinal thickness asymmetry in healthy Caucasian adults measured by SPECTRALIS SD-OCT posterior pole analysis. Invest Ophthalmol Vis Sci., 56(6): 3875- 3882.

7. Rajendrababu S, Gupta N, Vijayakumar B et al. (2014): Screening First Degree Relatives of Persons with Primary Open Angle Glaucoma in India. J Curr Glaucoma Pract., 8(3):107-112.
8. Micheal S, Humaira A, Khan M et al. (2014): Association of known common genetic variants with primary open angle, primary angle closure, and pseudoexfoliation glaucoma in Pakistani cohorts. Mol Vis., 20:1471-1479.

9. Mantravadi AV, Vadhar N (2015): Glaucoma. Prim Care, 42(3):437-449.

10. Renard JP, Rouland JF, Bron A et al. (2013): Nutritional, lifestyle and environmental factors in ocular hypertension and primary open-angle glaucoma: an exploratory case-control study. Acta Ophthalmol., 91(6): 505-513.

11. Park S, Jamshidi Y, Vaideanu D et al. (2009): Genetic Risk for Primary Open-Angle Glaucoma Determined by LMX1B Haplotypes. Invest Opthalmol Vis Sci., 50(4):1522-1530.

12. Khanal S, Davey PG, Racette $L$ et al. (2016):Intraeye retinal nerve fiber layer and macular thickness asymmetry measurements for the discrimination of primary open-angle glaucoma and normal tension glaucoma. J Optom., 9(2):118125.

13. Medeiros FA, Zangwill LM, Alencar LM et al. (2009): Detection of glaucoma progression with stratus OCT Retinal Nerve Fiber Layer, Optic Nerve Head, and Macular Thickness Measurements. Invest Opthalmol Vis Sci., 50(12):5741-5748.

14. Parikh RS, Parikh SR, Thomas R (2010): Diagnostic capability of macular parameters of Stratus OCT 3 in detection of early glaucoma. Br J Opthalmol., 94(2): 197-201.

15. Wollstein G, Ishikawa $\mathbf{H}$, Wang $\mathbf{J}$ et al. (2005): Comparison of three optical coherence tomography scanning areas for detection of glaucomatous damage. Am J Opthamol., 139(1):3943.

16. Sullivan-Mee M, Ruegg CC, Pensyl D et al. (2013): Diagnostic Precision of Retinal Nerve Fiber Layer and Macular Thickness Asymmetry Parameters for Identifying Early Primary OpenAngle Glaucoma. Am J Opthalmol.,156(3):567-577.

17. Um TW, Sung KR, Wollstein G et al. (2012): Asymmetry in Hemifield Macular Thickness as an Early Indicator of Glaucomatous Change. Invest Ophthalmol Vis Sci., 53(3):11391144.

18. Yamashita T, Sakamoto T, Kakiuchi N et al. (2014): Posterior Pole Asymmetry Analyses of Retinal Thickness of Upper and Lower Sectors and Their Association with Peak Retinal Nerve Fiber Layer Thickness in Healthy Young Eyes. Invest Ophthalmol Vis Sci., 55(9):5673-5678.

19. Yang $Z$, Tatham AJ, Weinreb RN et al. (2015): Diagnostic Ability of Macular Ganglion Cell Inner Plexiform Layer Measurements in Glaucoma Using Swept Source and Spectral Domain Optical Coherence Tomography. PLoS ONE, 10(5): e0125957.

20. Rolle T, Dallorto L, Briamonte C et al. (2014): Retinal nerve fiber layer and macular thickness analysis with Fourier domain optical coherence tomography in subjects with a positive family history for primary open angle glaucoma. Br J Opthalmol., 98(9):1240-1244.

21. Green CM, Kearns LS, Wu J et al. (2007): How significant is a family history of glaucoma? Experience from the Glaucoma Inheritance Study in Tasmania. Clin Exp Opthalmol., 35(9):793799.

22. Kong X, Zhu W, Chen $X$ et al. (2013): Familial aggregation of primary open angle glaucoma in Shanghai, China. Mol Vis.,19: 1859-1865. 